Journal of Mathematical Physics, Analysis, Geometry

2015, vol. 11, No. 3, pp. 267-278

\title{
Bimodal Distributions in the Space of a Non-Uniform Weight
}

\author{
N.V. Lemesheva \\ Department of Mechanics and Mathematics, V.N. Karazin Kharkiv National University \\ 4 Svobody Sq., Kharkiv 6107r, Ukraine \\ E-mail: lemesheva.kharkov@rambler.ru
}

Received December 16, 2014, revised May 13, 2015

\begin{abstract}
Bimodal approximate solutions of the Boltzmann equation for the model of hard spheres, which describe the interaction between the "accelerationpacking" flows, are constructed. Some sufficient conditions for infinitesimality of the mixed error "with weight" between the left-hand and the right-hand sides of the equation are obtained.
\end{abstract}

Key words: Boltzmann equation, hard spheres, Maxwellian, bimodal distribution, approximate solution, error, non-uniform weight..

Mathematics Subject Classification 2010: 76P05, 45K05 (primary); 82C40, 35Q55 (secondary).

\section{Introduction}

The well-known equation of the kinetic theory of gases is a nonlinear integraldifferential Boltzmann equation, which in the case of hard spheres model is as follows [3]:

$$
\begin{gathered}
D(f)=Q(f, f) ; \\
D(f)=\frac{\partial f}{\partial t}+v \frac{\partial f}{\partial x} ; \\
Q(f, f)=\frac{d^{2}}{2} \int_{R^{3}} d v_{1} \int_{\Sigma} d \alpha\left|\left(v-v_{1}, \alpha\right)\right| \\
\times\left[f\left(t, v_{1}^{\prime}, x\right) f\left(t, v^{\prime}, x\right)-f\left(t, v_{1}, x\right) f(t, v, x)\right],
\end{gathered}
$$

describing the behavior of the molecular distribution function $f=f(t, v, x)$, where $x=\left(x^{1}, x^{2}, x^{3}\right) \in R^{3}, v=\left(v^{1}, v^{2}, v^{3}\right) \in R^{3}$ are the coordinate and the velocity of molecules at the moment of time $t \in R^{1}$, respectively; $\frac{\partial f}{\partial x}$ is the spatial 
gradient of the function, $d>0$ is the molecular diameter, $\alpha$ is the vector on the unit sphere $\Sigma$ in $R^{3} ; v, v_{1}$ and $v^{\prime}, v_{1}^{\prime}$ are the velocities of two molecules before and after the collision, respectively; and the law of collision of two hard spheres has the form

$$
\left\{\begin{array}{l}
v^{\prime}=v+\alpha\left(\alpha, v_{1}-v\right) \\
v_{1}^{\prime}=v_{1}-\alpha\left(\alpha, v_{1}-v\right)
\end{array}\right.
$$

The difficulties of solving this equation appear because the function $f=$ $f(t, v, x)$ is multidimensional and the right-hand side of equation (1)-(3) has a complex form. Currently, for the model of hard spheres only one exact solution of the Boltzmann equation in equilibrium state is known, namely, the Maxwell's distribution $[2,3]$ :

$$
M(t, v, x)=\rho\left(\frac{\beta}{\pi}\right)^{3 / 2} e^{-\beta(v-\widetilde{v})^{2}},
$$

where $\rho$ is the density, $\beta=\frac{1}{2 T}$ is the inverse temperature, $\widetilde{v}$ is the mass flow rate (in the case of $\widetilde{v}=$ const, Maxwellian is called global if $\widetilde{v}$ depends on $t$ and $x$ is the local Maxwellian). The physical meaning and the geometrical structure of such solutions were considered in detail in $[2,5,8,11,12]$ (for other models, especially Maxwell molecules, the non-Maxwellian exact solution, the so-called BWK-mode, was obtained in $[1,4,13])$.

In the description of a non-equilibrium process in the gas we can obtain only approximate solutions of the Boltzmann equation, which can be sought in the form of bimodal distributions, i.e., the linear combinations of two Maxwellians with different hydrodynamic parameters. These distributions are as follows $[14$, $15]$ :

$$
f=\varphi_{1} M_{1}+\varphi_{2} M_{2},
$$

where $M_{i}, i=1,2$ are Maxwellians of different types [6-10], $\varphi_{i}=\varphi_{i}(t, x) \geq 0$, $\varphi_{i} \in C^{1}\left(R^{4}\right), i=1,2$ are the coefficient functions which should be found such that the difference between the left-hand and the right-hand sides of equation (1) is arbitrarily small.

To build these bimodal distributions with different modes, which describe the interaction between flows in a gas of hard spheres and satisfy the Boltzmann equation with whatever degree of accuracy, we consider different norms of the difference $D(f)-Q(f, f)$ between the parts of equation (1) which we introduce below using some additional definitions.

Definition 1. We denote by $C L\left(R^{k} \times R^{m}\right)$ the space of the functions $g(y, z)$, $y \in R^{k}, z \in R^{m}$, where $k, m \geq 0$, with the norm

$$
\|g\|=\sup _{(t, x) \in R^{k}} \int_{R^{m}}|g(y, z)| d z .
$$


Definition 2. We denote by $\widetilde{C} L\left(R^{4} \times R^{3}\right)$ and $\widetilde{C}_{q} L\left(R^{4} \times R^{3}\right)$ the spaces of the function $g(t, v, x), t \in R^{1}, x \in R^{3}, v \in R^{3}$, with the norms

$$
\|g\|=\left\|\frac{g}{1+|t|}\right\|_{C L\left(R^{4} \times R^{3}\right)}=\sup _{(t, x) \in R^{4}} \frac{1}{1+|t|} \int_{R^{3}}|g(t, x, v)| d v
$$

and

$$
\|g\|=\left\|\frac{g q(x)}{1+|t|}\right\|_{C L\left(R^{4} \times R^{3}\right)}=\sup _{(t, x) \in R^{4}} \frac{q(x)}{1+|t|} \int_{R^{3}}|g(t, x, v)| d v,
$$

respectively. The function $q(x) \geq 0$ is bounded on $R^{3}$.

Definition 3. We will call the uniform integral ("mixed") error, the error with a "uniform weight" and the error with a "non-uniform weight" for the Boltzmann equation, the following quantities:

$$
\begin{aligned}
\Delta & =\|D(f)-Q(f, f)\|_{C L\left(R^{4} \times R^{3}\right)}, \\
\widetilde{\Delta} & =\|D(f)-Q(f, f)\|_{\widetilde{C} L\left(R^{4} \times R^{3}\right)}, \\
\widetilde{\Delta}_{q} & =\|D(f)-Q(f, f)\|_{\widetilde{C}_{q} L\left(R^{4} \times R^{3}\right)},
\end{aligned}
$$

respectively.

The description of the transient behavior between the flows of "acceleratingpacking" type, obtained by building a bimodal distribution form (5) with different coefficient functions with the uniformly integrable error $\Delta$ and the "mixed weighted" error $\widetilde{\Delta}$, was given in $[9,10]$.

The aim of the paper is to study the behavior of the error with a "non-uniform weight" $\widetilde{\Delta}_{q}$ for bimodal distributions (considered in [10]) describing the interaction between the two Maxwell flows of hard spheres of "accelerating-packing" type with time-dependent density, which allow us to get the results different from those obtained in the previous work [10].

\section{Statement of the Problem}

We have to find a bimodal distribution $f$ in the form (5), where $\varphi_{i}=\varphi_{i}(t, x)$ are bounded on $R^{4}$ together with their derivatives, and the Maxwellians $M_{i}$ represent the flows of "accelerating-packing" type [8-10]:

$$
\begin{gathered}
M_{i}=\rho_{i}\left(\frac{\beta_{i}}{\pi}\right)^{3 / 2} e^{-\beta_{i}\left(v-\widetilde{v}_{i}\right)^{2}}, i=1,2, \\
\rho_{i}=\bar{\rho}_{i} e^{\beta_{i}\left(\widetilde{v}_{i}^{2}+2 \bar{u}_{i} x\right)}, i=1,2,
\end{gathered}
$$




$$
\widetilde{v}_{i}=\bar{v}_{i}-\bar{u}_{i} t, i=1,2,
$$

where $\bar{\rho}_{i}, \bar{v}_{i}, \bar{u}_{i}$ are the scalar and the vector constants, $\beta_{i}=\frac{1}{2 T_{i}}$ are the inverse temperatures, $\widetilde{v}_{i}=\widetilde{v}_{i}(t)$ is the mass flow rate, $\rho_{i}=\rho_{i}(t, x)$ is the density of $i$-flow rate.

We have to find the functions $\varphi_{i}, i=1,2$ and the behavior of all available parameters such that the uniform integral error with a "non-uniform weight"

$$
\widetilde{\Delta}_{q}=\sup _{(t, x) \in R^{4}} \frac{q(x)}{1+|t|} \int_{R^{3}}|D(f)-Q(f, f)| d v,
$$

where $q(x)$ is the non-negative bounded function on $R^{3}$, is arbitrarily small.

Some solutions of this problem are given below.

\section{Main Results}

Theorem 1. Let in a bimodal distribution (5) with the modes $M_{i}$ of type (9)-(11) the functions $\varphi_{i}, i=1,2$ not depend on $\beta_{i}, i=1,2$ and have the form

$$
\varphi_{i}(t, x)=C_{i}\left(x+\bar{u}_{i} \frac{\left(\bar{v}_{i}-\bar{u}_{i} t\right)^{2}}{2 \bar{u}_{i}^{2}}\right), i=1,2,
$$

where the smooth functions $C_{i} \geq 0$ are bounded on $R^{3}$ together with their derivatives.

Suppose that

$$
\begin{array}{ll}
\bar{u}_{i}=\bar{u}_{o i} \beta_{i}^{-n_{i}}, & i=1,2, \\
\bar{v}_{i}=\bar{v}_{o i} \beta_{i}^{-k_{i}}, & i=1,2,
\end{array}
$$

where $\bar{u}_{o i}, \bar{v}_{o i} \in R^{3}$ are arbitrary fixed vectors and $n_{i}, k_{i}$ are such that

$$
n_{i} \geq 1 ; k_{i} \geq \frac{1}{2} ; k_{i} \geq \frac{n_{i}}{2}, \quad i=1,2 .
$$

Suppose also that the function $q(x)$ is such that the value of $q(x) e^{2 \bar{u}_{o i} x}$ is bounded on $R^{3}$.

Then the following statement is true:

$$
\lim _{\substack{\beta_{1} \rightarrow+\infty \\ \beta_{2} \rightarrow+\infty}} \widetilde{\Delta}_{q}=0
$$


P r o o f. By substituting (5) into (2) and (3), and then into (12), taking into consideration $(9)-(11)$, and using the technique developed in $[9,10]$, we get the estimation $\widetilde{\Delta}_{q}^{\prime}$ for the error $\widetilde{\Delta}_{q}$ :

$$
\begin{aligned}
\widetilde{\Delta}_{q} \leq \widetilde{\Delta}_{q}^{\prime} & =\sup _{(t, x) \in R^{4}} \frac{q(x)}{1+|t|} \sum_{i, j=1, i \neq j}^{2}\left[\int_{R^{3}} \mid \frac{\partial \varphi_{i}}{\partial t}+\left(\frac{u}{\sqrt{\beta_{i}}}+\bar{v}_{i}-\bar{u}_{i} t\right) \frac{\partial \varphi_{i}}{\partial x}\right. \\
& +\varphi_{1} \varphi_{2} \rho_{j}(t, x) \frac{d^{2}}{\sqrt{\pi}} \int_{R^{3}} F_{i j} e^{-w^{2}} d w \mid \rho_{i}(t, x) \pi^{-3 / 2} e^{-u^{2}} d u \\
& \left.+\varphi_{1} \varphi_{2} \frac{\rho_{1}(t, x) \rho_{2}(t, x)}{\pi^{2}} d^{2} \int_{R^{6}} e^{-w^{2}-u^{2}} F_{i j} d w d u\right],
\end{aligned}
$$

where

$$
F_{i j}=\left|\frac{u}{\sqrt{\beta_{i}}}+\bar{v}_{i}-\bar{v}_{j}+\left(\bar{u}_{j}-\bar{u}_{i}\right) t-\frac{w}{\sqrt{\beta_{j}}}\right|, \quad i, j=1,2, i \neq j
$$

and

$$
\rho_{i}(t, x)=\bar{\rho}_{i} \exp \left\{\beta_{i}\left(\left(\bar{v}_{i}-\bar{u}_{i} t\right)^{2}+2 \bar{u}_{i} x\right)\right\}, i=1,2 .
$$

The correctness of this estimation is conditioned by the fact that, in view of (13) and (20), the values

$$
t \varphi_{i} \rho_{i}(t, x) ; \frac{\partial \varphi_{i}}{\partial t} \rho_{i}(t, x) ;\left|\frac{\partial \varphi_{i}}{\partial x}\right| \rho_{i}(t, x) ; t\left(\bar{u}_{i}, \frac{\partial \varphi_{i}}{\partial x}\right) \rho_{i}(t, x), \quad i=1,2
$$

are bounded on $R^{4}$ after multiplication by $\frac{q(x)}{1+|t|}$.

Since $\varphi_{i}$ is the smooth function, the values $\sqrt{|t|} \varphi_{i} \rho_{i}(t, x), i=1,2$ and $|t| \varphi_{1} \varphi_{2} \rho_{1}(t, x) \rho_{2}(t, x)$ are bounded either with the factor $|t|$ or without it.

The transition to the limit for $\beta_{1}, \beta_{2} \rightarrow+\infty$ in (13), (18)-(20), with (14)(16) being taken into account, is analogous to the transition described in [10] and gives the same result (the possibility of the transition marked by suprema and integrals is justified in the same way as in $[9,10]$ by using Lemma 1 from [7]):

$$
\begin{array}{r}
\lim _{\substack{\beta_{1} \rightarrow+\infty \\
\beta_{2} \rightarrow+\infty}} \rho_{i}(t, x)=\bar{\rho}_{i} \mu_{i}(x)=\left[\begin{array}{ll}
\bar{\rho}_{i} ; & n_{i}>1 ; k_{i}>\frac{1}{2}, i=1,2, \\
\bar{\rho}_{i} e^{2 \bar{o}_{o i} x} ; & n_{i}=1 ; k_{i}>\frac{1}{2}, i=1,2, \\
\bar{\rho}_{i} e^{\bar{v}_{o i}^{2}+2 \bar{u}_{o i} x} ; & n_{i}=1 ; k_{i}=\frac{1}{2}, i=1,2 ;
\end{array}\right. \\
\lim _{\substack{\beta_{1} \rightarrow+\infty \\
\beta_{2} \rightarrow+\infty}} \varphi_{i}=C_{i}\left(x+a_{i}\right)=\left[\begin{array}{ll}
C_{i}(x), & k_{i}>\frac{1}{2} n_{i}, i=1,2, \\
C_{i}\left(x+\frac{\bar{u}_{o i} \bar{o}_{0 i}^{2}}{2 \bar{u}_{o i}^{2}}\right), & k_{i}=\frac{1}{2} n_{i}, i=1,2 ;
\end{array}\right.
\end{array}
$$




$$
\lim _{\substack{\beta_{1} \rightarrow+\infty \\ \beta_{2} \rightarrow+\infty}} \frac{\partial \varphi_{i}}{\partial t}=0 ; \lim _{\substack{\beta_{1} \rightarrow+\infty \\ \beta_{2} \rightarrow+\infty}} \frac{\partial \varphi_{i}}{\partial x}=C_{i}^{\prime}\left(x+a_{i}\right) ; \lim _{\substack{\beta_{1} \rightarrow+\infty \\ \beta_{2} \rightarrow+\infty}} F_{i j}=0, i \neq j .
$$

Consequently,

$$
\lim _{\substack{\beta_{1} \rightarrow+\infty \\ \beta_{2} \rightarrow+\infty}} \widetilde{\Delta}_{q}^{\prime}=0
$$

Hence, by (18) we obtain (17). The theorem is proved.

$\mathrm{R}$ e $\mathrm{m}$ a r k 1 . As it can be seen from (20), (22)-(24), the coefficients of the functions $C_{i}, i=1,2$, in (18) increase only along the vector $\bar{u}_{i}$. Thus, the condition of the finiteness or rapid decay for the functions $C_{i}$ (imposed in [10]) in this paper is weakened because the function $q(x)$ is bounded. However, $q(x)$ can be not only bounded but also finite or rapidly decreasing at least in the directions parallel to the vector $\bar{u}_{o i}$.

Theorem 2. Let the functions $\varphi_{i}$ in distribution (5) have the form:

$$
\varphi_{i}(t, x)=\psi_{i}(t, x) \exp \left\{-\beta_{i}\left(\left(\bar{v}_{i}-\bar{u}_{i} t\right)^{2}+2 \bar{u}_{i} x\right)\right\}, \quad i=1,2,
$$

and each of the products of multiplication of $\frac{q(x)}{1+|t|}$ by the functions

$$
t \psi_{1} \psi_{2} ; \frac{\partial \psi_{i}}{\partial t} ; t \psi_{i} ;\left|\frac{\partial \psi_{i}}{\partial x}\right| ; t\left(\bar{u}_{i}, \frac{\partial \psi_{i}}{\partial x}\right), \quad i=1,2
$$

be bounded by $t$ and $x$ on $R^{4}$. Suppose, besides, condition (14) is fulfilled for

$$
n_{i}>\frac{1}{2}
$$

Then there is the value $\widetilde{\Delta}_{q}^{\prime}$ such that

$$
\widetilde{\Delta}_{q} \leq \widetilde{\Delta}_{q}^{\prime}
$$

and

$$
\begin{array}{r}
\lim _{\beta_{1}, \beta_{2} \rightarrow+\infty} \widetilde{\Delta}_{q}^{\prime}=\sup _{(t, x) \in R^{4}} \frac{q(x)}{1+|t|} \sum_{i, j=1, i \neq j}^{2} \bar{\rho}_{i} \mid \frac{\partial \psi_{i}}{\partial t}+\bar{v}_{i} \frac{\partial \psi_{i}}{\partial x}+ \\
+\psi_{1} \psi_{2} \pi d^{2} \bar{\rho}_{j}\left|\bar{v}_{1}-\bar{v}_{2}\right|\left|+2 \pi d^{2} \bar{\rho}_{1} \bar{\rho}_{2}\right| \bar{v}_{1}-\bar{v}_{2} \mid \sup _{(t, x) \in R^{4}} \frac{q(x)}{1+|t|}\left(\psi_{1} \psi_{2}\right) .
\end{array}
$$


P r o o f. The derivative of the functions $\varphi_{i}$, accounting (20) and (26), will be as follows:

$$
\begin{gathered}
\frac{\partial \varphi_{i}}{\partial t}=\bar{\rho}_{i}\left[\rho_{i}(t, x)\right]^{-1}\left\{\frac{\partial \psi_{i}}{\partial t}+2 \beta_{i} \psi_{i}\left(\left(\bar{v}_{i}, \bar{u}_{i}\right)-t \bar{u}_{i}^{2}\right)\right\}, \quad i=1,2, \\
\frac{\partial \varphi_{i}}{\partial x}=\bar{\rho}_{i}\left[\rho_{i}(t, x)\right]^{-1}\left\{\frac{\partial \psi_{i}}{\partial x}-2 \beta_{i} \psi_{i} \bar{u}_{i}\right\}, \quad i=1,2 .
\end{gathered}
$$

Condition (27) guarantees the existence of the suprema over $t$ and $x$ and thus, in view of (26), instead of (18), we obtain

$$
\widetilde{\Delta}_{q}^{\prime}=\pi^{-3 / 2} \sup _{(t, x) \in R^{4}} \frac{q(x)}{1+|t|} \sum_{i=1}^{2} \bar{\rho}_{i} \int_{R^{3}}\left[\left|\frac{\partial \psi_{i}}{\partial t}+A_{i}+B_{i}\right|+A_{i}\right] e^{-u^{2}} d u
$$

where

$$
\begin{gathered}
A_{i}=A_{i}(u, t)=\psi_{1} \psi_{2} \frac{d^{2}}{\sqrt{\pi}} \bar{\rho}_{j} \int_{R^{3}} e^{-w^{2}} F_{i j} d w, \quad i \neq j, \\
B_{i}=B_{i}(u, t)=\frac{\partial \psi_{i}}{\partial x}\left(\frac{u}{\sqrt{\beta_{i}}}+\bar{v}_{i}-\bar{u}_{i} t\right)-2 \psi_{i} \sqrt{\beta_{i}}\left(u, \bar{u}_{i}\right)
\end{gathered}
$$

with (19) retained.

Due to conditions (14) and (28), after transition to the limit in (34), (35) and (19), by estimating (33), we obtain

$$
\begin{array}{r}
\lim _{\substack{\beta_{1} \rightarrow+\infty \\
\beta_{2} \rightarrow+\infty}} \widetilde{\Delta}_{q}^{\prime}=\pi^{-3 / 2} \sup _{(t, x) \in R^{4}} \frac{q(x)}{1+|t|} \sum_{i, j=1, i \neq j}^{2} \bar{\rho}_{i} \int_{R^{3}}\left[\mid \frac{\partial \psi_{i}}{\partial t}\right. \\
\left.+\psi_{1} \psi_{2} \pi d^{2} \bar{\rho}_{j}\left|\bar{v}_{1}-\bar{v}_{2}\right|+\bar{v}_{i} \frac{\partial \psi_{i}}{\partial x}\left|+\psi_{1} \psi_{2} \pi d^{2} \bar{\rho}_{j}\right| \bar{v}_{1}-\bar{v}_{2} \mid\right] e^{-u^{2}} d u
\end{array}
$$

which, after integration with respect to $u$, leads to (30). The theorem is proved.

One of possible sufficient conditions for arbitrarily small error (12) is provided in the corollary below.

Corollary 1. Let all the conditions of Theorem 2 be valid, and the functions $\psi_{i}$ be of the form

$$
\psi_{i}=C_{i}\left(x-\bar{v}_{i} t\right), \quad i=1,2
$$

or

$$
\psi_{i}=C_{i}\left(\left[x \times \bar{v}_{i}\right]\right), \quad i=1,2 .
$$

Then (17) holds true if the following conditions are fulfilled:

1) $C_{i} \geq 0$ are bounded smooth functions, 
2) the products of $q(x) C_{i}$ and $q(x) C_{i}^{\prime}$ are bounded,

3)

$$
\bar{v}_{1}=\bar{v}_{2}
$$

$\mathrm{P} \mathrm{r}$ o o $\mathrm{f}$. Let us find the derivative of both functions with respect to $t$ and $x$. For the case (37), we get

$$
\frac{\partial \psi_{i}}{\partial x}=C_{i}^{\prime} ; \quad \frac{\partial \psi_{i}}{\partial t}=-\left(\bar{v}_{i}, C_{i}^{\prime}\right), \quad i=1,2,
$$

and for the case (38),

$$
\frac{\partial \psi_{i}}{\partial x}=\left[\bar{v}_{i} \times C_{i}^{\prime}\right] ; \quad \frac{\partial \psi_{i}}{\partial t}=0, \quad i=1,2 .
$$

We can see that in both cases we have

$$
\frac{\partial \psi_{i}}{\partial t}+\bar{v}_{i} \frac{\partial \psi_{i}}{\partial x}=0, \quad i=1,2
$$

Statement (30) is true, as the functions of the forms (37) and (38) satisfy the conditions of Theorem 2 if the conditions of this corollary are fulfilled.

Hence, using (39), we obtain (17). The corollary is proved.

Theorem 3. Let

$$
\varphi_{i}(t, x)=\psi_{i}(t, x) \exp \left\{-\beta_{i}\left(\bar{v}_{i}-\bar{u}_{i} t\right)^{2}\right\}, \quad i=1,2,
$$

where each product of multiplication of $\exp \left\{2 \beta_{i} \bar{u}_{i} x\right\}$ by the functions of the form (27) is bounded with the weight $\frac{q(x)}{1+|t|}$.

Let also condition (14) take place for $n_{i} \underset{\widetilde{\sim}}{\gtrless}$.

Then there is a finite limit to the value $\widetilde{\Delta}_{q}^{\prime}$, and (29) is true such that:

1) if $n_{i}>1$, then the estimation $\widetilde{\Delta}_{q}^{\prime}$ for the error $\widetilde{\Delta}_{q}$ is equal to (30);

2) if $n_{i}=1$, then

$$
\begin{array}{r}
\lim _{\substack{\beta_{1} \rightarrow+\infty \\
\beta_{2} \rightarrow+\infty}} \widetilde{\Delta}_{q}^{\prime}=\sum_{i, j=1, i \neq j}^{2} \bar{\rho}_{i} \sup _{(t, x) \in R^{4}} \frac{q(x)}{1+|t|} \mid e^{2 \bar{u}_{o i} x}\left(\frac{\partial \psi_{i}}{\partial t}+\bar{v}_{i} \frac{\partial \psi_{i}}{\partial x}\right) \\
+\psi_{1} \psi_{2} e^{2 \bar{u}_{o 1} x} e^{2 \bar{u}_{o 2} x} \cdot \pi d^{2} \bar{\rho}_{j}\left|\bar{v}_{1}-\bar{v}_{2}\right| \mid \\
+2 \pi d^{2} \bar{\rho}_{1} \bar{\rho}_{2}\left|\bar{v}_{1}-\bar{v}_{2}\right| \sup _{(t, x) \in R^{4}} \frac{q(x)}{1+|t|}\left[e^{2 \bar{u}_{o 1} x} e^{2 \bar{u}_{o 2} x} \psi_{1}(t, x) \psi_{2}(t, x)\right] \\
+2 \sum_{i=1}^{2} \bar{\rho}_{i}\left|\left(\bar{u}_{o i}, \bar{v}_{i}\right)\right| \sup _{(t, x) \in R^{4}} \frac{q(x)}{1+|t|}\left\{e^{2 \bar{u}_{o i} x} \psi_{i}(t, x)\right\} .
\end{array}
$$


$\mathrm{P} \mathrm{r} \mathrm{o} \mathrm{o} \mathrm{f.} \mathrm{The} \mathrm{proof} \mathrm{follows} \mathrm{the} \mathrm{same} \mathrm{pattern} \mathrm{as} \mathrm{the} \mathrm{proof} \mathrm{of} \mathrm{Theorem} 3$ in [10]. Having carried out a similar transformation, but for the error (12), and passing to the limit in (34) and (35) for $\beta_{1}, \beta_{2} \rightarrow+\infty$, we find:

$$
\begin{gathered}
\lim _{\substack{\beta_{1} \rightarrow+\infty \\
\beta_{2} \rightarrow+\infty}} A_{i}=\left[\begin{array}{c}
\psi_{1} \psi_{2} \pi d^{2} \bar{\rho}_{j}\left|\bar{v}_{1}-\bar{v}_{2}\right|, n_{i}>1, \\
e^{2 \bar{u}_{o i} x} \psi_{1} \psi_{2} \pi d^{2} \bar{\rho}_{j}\left|\bar{v}_{1}-\bar{v}_{2}\right|, n_{i}=1, i \neq j,
\end{array} \quad i, j=1,2 ;\right. \\
\lim _{\substack{\beta_{1} \rightarrow+\infty \\
\beta_{2} \rightarrow+\infty}} B_{i}=\left[\begin{array}{c}
\bar{v}_{i} \frac{\partial \psi_{i}}{\partial x}, n_{i}>1, \\
\bar{v}_{i} \frac{\partial \psi_{i}}{\partial x}+2 \psi_{i}\left(\bar{u}_{o i}, \bar{v}_{i}\right), n_{i}=1,
\end{array} \quad i=1,2 .\right.
\end{gathered}
$$

Further, substituting (44) and (45) into (30), we obtain:

1) for $n_{i}>1, \lim _{\substack{\beta_{1} \rightarrow+\infty \\ \beta_{2} \rightarrow+\infty}} \widetilde{\Delta}_{q}^{\prime}$ coincides with (30) (since the limit value $e^{2 \beta_{i} \bar{u}_{i} x}$ is equal to 1 );

2) for $n_{i}=1, \lim _{\substack{\beta_{1} \rightarrow+\infty \\ \beta_{2} \rightarrow+\infty}} \widetilde{\Delta}_{q}^{\prime}$ has the form (43) (here $\lim _{\substack{\beta_{1} \rightarrow+\infty \\ \beta_{2} \rightarrow+\infty}} e^{2 \beta_{i} \bar{u}_{i} x}=e^{2 \bar{u}_{o i} x}$, which causes the appearance of an additional multiplier and amount when we pass to the limit for $\widetilde{\Delta}_{q}^{\prime}$ ).

The theorem is proved.

Corollary 2. Let the parameters $n_{i}, i=1,2$ in (14) satisfy $n_{i}>1$. In addition, let the functions $\psi_{i}$ have the form (37) or (38) and let either of the following conditions:

1)

$$
\operatorname{supp} C_{1} \cap \operatorname{supp} C_{2}=\oslash
$$

or

$$
\bar{v}_{1}=\bar{v}_{2},
$$

where $C_{i}$ and $C_{i}^{\prime}$ are bounded functions;

2) the function $q(x)$ is finite or fast decreasing at infinity,

be fulfilled.

Then for any $\bar{u}_{i}, \bar{v}_{i}$ and $\varphi_{i}$ of the form (42), (17) is true.

P r o o f. We verify that the functions of the forms (37) and (38) satisfy the conditions of Theorem 3 if the conditions of this corollary are fulfilled. First, make sure that the following quantities are bounded:

$$
\frac{q(x)}{1+|t|} t \psi_{1} \psi_{2} \exp \left\{2 \beta_{1} \bar{u}_{1} x+2 \beta_{2} \bar{u}_{2} x\right\}, \quad i=1,2 ;
$$




$$
\begin{gathered}
\frac{q(x)}{1+|t|} \frac{\partial \psi_{i}}{\partial t} \exp \left\{2 \beta_{i} \bar{u}_{i} x\right\}, \quad i=1,2 ; \\
\frac{q(x)}{1+|t|} t \psi_{i} \exp \left\{2 \beta_{i} \bar{u}_{i} x\right\}, \quad i=1,2 ; \\
\frac{q(x)}{1+|t|}\left|\frac{\partial \psi_{i}}{\partial x}\right| \exp \left\{2 \beta_{i} \bar{u}_{i} x\right\}, \quad i=1,2 ; \\
\frac{q(x)}{1+|t|} t\left(\bar{u}_{i}, \frac{\partial \psi_{i}}{\partial x}\right) \exp \left\{2 \beta_{i} \bar{u}_{i} x\right\}, \quad i=1,2 .
\end{gathered}
$$

It is easily seen that for both functions of the forms (37) and (38), quantity (48) is the same, and under condition (46) is identically equal to zero. Under condition (47), quantity (48) is bounded because the functions $C_{i}$ are bounded and the function $q(x)$ is finite or fast decreasing, regardless of the structure of the argument (it is obvious that each of the multipliers $\frac{t}{1+|t|} ; C_{1} C_{2} ; q(x) \exp \left\{2 \beta_{1} \bar{u}_{1} x+2 \beta_{2} \bar{u}_{2} x\right\}$ is bounded).

Further, if conditions (46) and (47) are fulfilled, then (49) is zero when function (38) is used and is bounded by the conditions 1) and 2) of this corollary when function (37) is used.

Quantities (50)-(52) are evaluated in a similar manner using formulas (40) and (41). Consequently, we can use Theorem 3 for $n_{i}>1$, namely, formula (30). At the same time, taking into account (46) and (47), we find that $\lim _{\substack{\beta_{1} \rightarrow+\infty \\ \beta_{2} \rightarrow+\infty}} \widetilde{\Delta}_{q}^{\prime}=0$. Hence, the estimation for $\widetilde{\Delta}_{q}^{\prime}$ being well-defined in (29) for the error (12), we obtain (17). Corollary 2 is proved.

Corollary 3. Let all the conditions of Corollary 2 be fulfilled, but $n_{i}=1$. Suppose that the additional condition

$$
\left(\bar{u}_{i}, \bar{v}_{i}\right)=0, \quad i=1,2,
$$

is fulfilled.

Then the assertion of Corollary 2 remains true.

P r o o f. Since the functions of the forms (37) and (38) satisfy the conditions of Theorem 3 (which was proved in Corollary 2), we can use the second statement of the theorem, namely, formula (43).

The finiteness or sufficiently fast decreasing of the function $q(x)$ provides the boundedness of all members contained in (43) and suppresses the growth multiplier $e^{2 \bar{u}_{o i} x}, i=1,2$.

Considering conditions (46), (47) and (53), we come to the conclusion that all terms in (43) are equal to zero, and hence we again obtain (17). Corollary 3 is proved. 
$\mathrm{R}$ e $\mathrm{m}$ a $\mathrm{rk} 2$. Let us analyze the results. Since the error $\widetilde{\Delta}$, unlike the error $\widetilde{\Delta}_{q}$ used in the previous work [10], contains an additional factor $q(x)$, with the help of which it was possible to weaken the conditions imposed on the function $C_{i}$ (see Remark 1), then, from the viewpoint of the physical meaning, we can consider not only the "clumps", described by relation (37), and the "cylinders" of the form (38), but some flows in the whole space thus extending the class of the solutions.

Also, the function $q(x)$ in Corollary 1 can increase both the functions $C_{i}$ and their derivatives due to its sufficiently fast decreasing even along the vector $\bar{u}_{o i}$. There is no need to further minimize the error in $d \rightarrow 0$ for any $\bar{v}_{i}$, which was used in Corollary 1 of [10].

At the same time, by using a new error, it is possible to exclude the condition of perpendicularity of the vectors $\bar{u}_{i}$ and $\bar{v}_{i}$ used in Corollary 2 of [10] (for the vanishing of the functions $C_{i}$ in the directions in $R^{3}$, where the exponents in (45)-(49) increase) at least for the values of the parameter $n_{i}>1$, which allows us to generalize the results obtained for the parameters of a more general form than (14), (15).

\section{References}

[1] A.V. Bobylev, On the Exact Solutions of the Boltzmann Equation. - Dokl. Akad. Sci. of USSR. 225 (1975), No. 6, 1296-1299. (Russian)

[2] T. Carleman, Problemes Mathematiques dans la Theorie Cinetique des Gas. Almqvist and Wiksells, Uppsala, 1957.

[3] C. Cercignani, The Boltzmann Equation and its Applications. Springer, New York, 1988.

[4] M.H. Ernst, Exact Solution of the Non-Linear Boltzmann Equation for Maxwell Models. - Phys. Lett.A, 69 (1979), No.6, 390-392.

[5] O.G. Fridlender, Local Maxwell Solutions of the Boltzmann Equation. - J. Appl. Math. Mech. 29 (1965), No. 5, 973-977. (Russian)

[6] V.D. Gordevskii, An Approximate Biflow Solution of the Boltzmann Equation. Theoret. Math. Phys. (1998), No. 114, 126-136. (Russian)

[7] V.D. Gordevskyy, Biflow Distributions with Screw Modes. - Teoret. Math. Phys. 126 (2001), No. 2, 283-300. (Russian)

[8] V.D. Gordevskyy, On the Non-Stationary Maxwellians. - Math. Meth. Appl. Sci. 27 (2004), No. 2, 231-247.

[9] V.D. Gordevskyy and N.V. Andriyasheva, Interection between "AcceleratingPacking" Flows in a Low-Temperature Gas. - Math. Phys., Anal., Geom. 5 (2009), No. $1,38-53$. 
[10] V.D. Gordevskyy and N.V. Lemesheva, Transitional Regime between the Flows of "Accelerating-Packing" Type. - Bulletin of the V.N. Karazin Kharkiv National University, Ser. Math., Appl. Math., Mech. 931 (2010), 49-58. (Ukrainian)

[11] H. Grad, Principles of the Kinetic Theory of Gases, Handbuch der Physik, SpringerVerlag, Berlin 12 (1958), 205-294.

[12] M.N. Kogan, The Dynamics of a Rarefied Gas. Nauka, Moscow, 1967. (Russian)

[13] M. Krook and T.T. Wu, Formation of Maxwellian Tails. - Phys. Rev. Lett. 36 (1977), No. 19, 1107-1109.

[14] H.M. Mott-Smith, The Solution of the Boltzmann Equation for a Shock Wave. Phys. Rev. 82 (1951), No. 6, 885-890.

[15] T.E. Tamm, On the Width of High-Intensity Shock Waves. - Proc. (Trudy) Lebedev Phys. Inst. 29 (1965), 231-241. (Russian) 